\title{
Transmission: A Telepresence Interface for Neural and Kinetic Interaction
}

\author{
Oliver Gingrich, Alain Renaud, Eugenia Emets, Zhidong Xiao
}

\section{A B S T R A C T}

Transmission is both a telepresence performance and a research project. As a real-time visualization tool, Transmission creates alternate representations of neural activity through sound and vision, investigating the effect of interaction on human consciousness. As a sonification project, it creates an immersive experience for two users: a soundscape created by the human mind and the influence of kinetic interaction. An electroencephalographic (EEG) headset interprets a user's neural activity. An Open Sound Control (OSC) script then translates this data into a real-time particle stream and sound environment at one end. A second user in a remote location modifies this stream in real time through body movement. Together they become a telematic musical interface-communicating through visual and sonic representation of their interactions.

\section{Introduction}

Transmission is the second project by London-based collective Analema Group, in a series of art and research projects on telepresence. Using neurological data to engage audiences, Transmission presents users with an audiovisual experience that reflects their affective bodily resonance to telepresence. With Transmission, we present an audiovisual interface for communication that translates both neurological processes and kinetic dynamics into a new form of interactivity.

The technical aim of Transmission is to create an infrastructure to sonify and visualize brain activity during telepresence interactions. Users explore their own thought processes and neural activity in real time. A user in a remote location influences and thus interacts with these visual and sonic representations. Through motion capture techniques, the remote participant can directly manipulate and modify the interpretation of his interlocutor's brain activity (Figure I). Juxtaposing an inner, subjective and an external, kinetic activity, the EEG provides users with feedback on the psychological effect of their interaction.

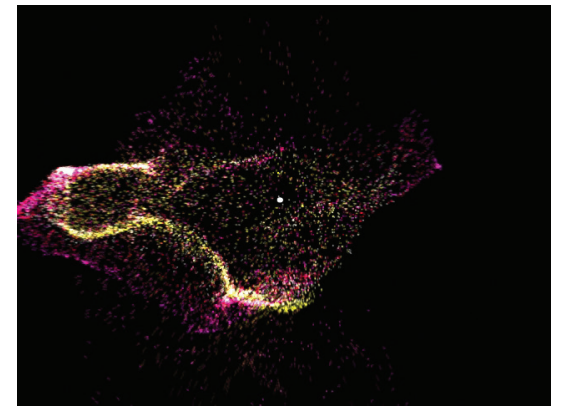

Figure 1. Analema Group, Transmission, 2014. Screenshot 1920 x 1080-Particle Test, December 2013. Representation of human brain activity changes in real time through EEG and kinetic data from a remote location. @ 2014 Analema Group.
Transmission creates an art form that constantly reinvents itself and lives through its participants and their respective communications. As research environment, the installation provides information on telepresence in the form of EEG data that are quantifiable and evaluated with the aid of standardized, qualitative tests. As artwork, Transmission is rooted in the history of telepresence art, interactive art, and audiovisual art.

\section{Concept}

Between the first use of the term "telepresence" by Marvin Minsky, in his article of the same name [I], and today stretch more than 30 years of academic and artistic discourse. Since Roy Ascott's pioneering work [2], a rich
Oliver Gingrich

CDE - Center for Digital Entertainment

Bournemouth University

Fern Barrow, Dorset, BH12 5BB

United Kingdom

oliver@musion.co.uk

Alain Renaud

CDE - Center for Digital Entertainment

Bournemouth University

Fern Barrow, Dorset, BH12 5BB

United Kingdom

arenaud@bournemouth.ac.uk

Eugenia Emets

Artist at Analema Group, London

126 Fernhead Road W9 3EN

London, United Kingdom

emetsjange@gmail.com

Zhidong Xiao

National Center for Computer Animation

Bournemouth University

Fern Barrow, Dorset, BH12 5BB

United Kingdom

zxiao@bournemouth.ac.uk 
history of telepresence art has evolved. Telepresence art developed along with the technologies it engages: higher bit and streaming rates, increasing processing and computing power, and optimized recording standards resulted in improved photorealism and enriched experiences.

Concurrent with technical developments, artistic and academic discourse developed along with the practice of its proponents. Paul Sermon's Telematic Dreaming [3], Telematic Séance [4], and Telematic Vision [5] focused on the ideas of remote presence and a sense of "being there." Tina Keane's experiments in telepresence art resulted in Couch, a telepresence environment exhibited all over the world [6]. The discourse shifted from an early concentration on translocation and disposition-for instance, in Rafael Lozano-Hemmer's The Trace [7] or Displaced Emperors [8] - to a concentration on virtuality [9] during the I990s. Since 2000, academics concentrated on posthumanism [Io] - for example in Stelarc and Olivero's performance Rotating Brains and Beating Heart [II]. Telepresence art is not confined to a single focus-it touches questions of a philosophical nature as much as questions of cultural identity and urbanism (for example, in Stanza's Connecting Cities [12]).

Over the past 30 years, the field has undergone an artistic, technological, and conceptual evolution. Artists have expanded their practice, outreach, and focus through the development and integration of new techniques, devices, and tools. New interfaces (Arduino, IR), new tools (such as motion capture, gesture control, and augmented reality), and new programming languages (processing, Max/MSP, OSC, VVVV, etc.) have augmented user experiences. Telepresence art continues to explore remote presence, boundaries of perception, and new forms of interactivity.

Interactive art and telepresence are strongly interrelated, both conceptually and technically. Ever since Marcel Duchamp's Bicycle Wheel [13], interactive art has been interested in user participation, chain reactions of cause and effect, and a kinetic discourse between art piece, author, and spectator. Whether users are invited to write with their eyes (as in Zach Lieberman's Eyewriter [14]) or physically experience rain (as in rAndom International's Rain Room [I5]), interactive arts tend to involve physical engagement as well as kinetic and bodily interactions.

Transmission not only stands in this tradition of visible and reactive movement, but also shifts the focus onto invisible dynamics - in this case generated by the user's brain through interaction. With Transmission, we widen the reach of telepresence from mere physical involvement to psychological engagement, from the conscious to the subconscious. Kinetic movement extends to our minds, our inner activities — an intangible dynamic of our respective, dislocated bodies. Contrary to traditional interactive arts, seen as going beyond psychological engagement [16], Transmission places interactive art in the realm of the subjective, psychological sphere of the self. The juxtaposition of physical and psychological interaction and neurological reaction builds the dialectic tension in Transmission.

Ultimately, with Transmission we are not only interested in exploring a sense of presence, of being elsewhere, but also in the effect of presence on a remote user. This piece is introspective as much as extroverted, looking at the effect presence has on our own minds. Interactive art doesn't have to be limited to a dialogue with technology or the other-Transmission focuses on interaction with the self (Figure 2).

Sound is the integral facilitator in this discourse between mind and body. Not only is sound foreboding, embedding, conditioning, and generative, it engenders visual representation. The user's brain waves are digitally modified to emphasize dominating dynamics. Certain neural 
affective states can be mapped to specific frequencies, sound bites, and sonic effects, thereby initiating empathic states. As a result, emotions are accentuated and thus easier to communicate to the remote user. The user's mind creates a soundscape for interaction-a piece of music of telepresence.

Since telepresence pioneer Pauline Oliveros founded her Deep Listening Institute [I7], sound artists have explored the relationship between telepresence and sound on multiple levels. Artistic engagement with neuroscience and music dates even further back: avant-garde artists and pioneers such as Yoko Ono and

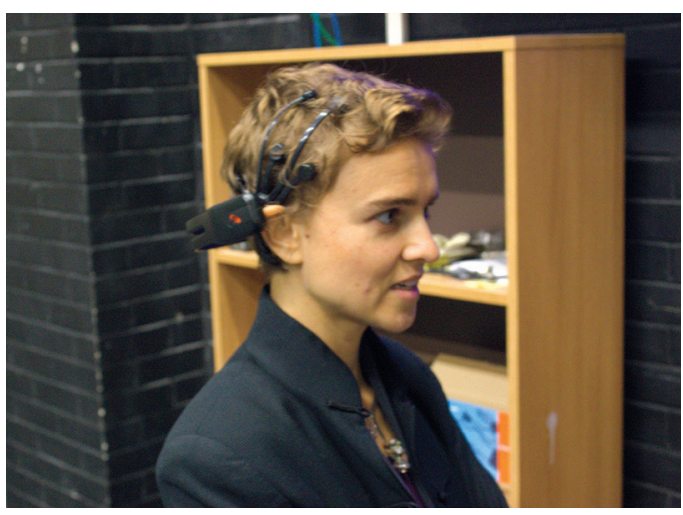

Figure 2. Eugenia Emets of Analema Group, Transmission EEG and sonification test. The EEG headset sends a real-time stream of data on affective states to an OSC script, allowing for realtime introspection of the user. Transmission looks at the effect of remote interaction on individuals. ( 2013 Analema Group. John Lennon experimented with EEGs and music as early as the 1960s. Alvin Lucier performed Music for Solo Performer at Brandeis University in 1965 [I8]. By making use of the headset as a wearable device, Lucier interpreted brain waves to generate soundscapes. A few years later, Richard Teitelbaum's Musica Elettronica Viva used EEG signals to manipulate electronic synthesizers [19]. In I97I, David Rosenboom's Ecology of the Skin created an orchestra of EEG music by working with a multitude of live participants [20]. As EEG headsets became more affordable, their use within the artistic and academic communities increased. Artists such as Eduardo Miranda [2I], Andrew Brouse (Biomusic) [22], or Marina Abramovic (The Magic of Mutual Gaze) [23] developed sophisticated patches for EEG interaction. Contemporary neurofeedback-inspired art takes all new forms: In 200I, Neam Cathode's Cyber Mondrian [24] incorporated EEG headsets into an audiovisual environment. Artist Mariko Mori's Wave UFO allows for an immersive brain sonification experience in which users can climb inside a spaceship [25]. Lisa Park's Eunoia maps brain activity to vibrations of water [26].

On a purely academic level, Jeffrey Thompson [27] studied the brain's reaction to specific frequencies. Sophisticated patches and interfaces for brain wave sonification have been developed, for instance by Mick Grierson at Goldsmith University. At the same time, whole departments have been dedicated to EEG and sound research: the Neuromusic Lab at the University of Plymouth, Stanford's Neuromusic Laboratory at the CCRMA, and departments at University of British Columbia and Technische Universitaet Graz.

Transmission is rooted in traditions of experimental sound, interactive arts, and telepresence art. The body's interactions, its extroverted bi-directionality between self and other, generate a sonic and visual imprint. Visual references for the design derive from ideas of connectedness, references to flight data visualizations, and Leonardo Da Vinci's representations of the human body as network (Figure 3). Transmission consists of a user experience, an art piece, and a research project. As the work is a cross-over between art and science, audiences learn to scrutinize telepresence experiences analytically by experiencing the effect in real time.

Transmission thus facilitates an understanding of the functioning of our inner landscape. Ethical questions about measuring emotions and linking these to visual representations-i.e. questions about the transparent human-are explored interactively.

On a secondary level, we aim for science to profit from new visualization and representation techniques by including experts in our discourse. With a neuroscientist (UCL London) and a 


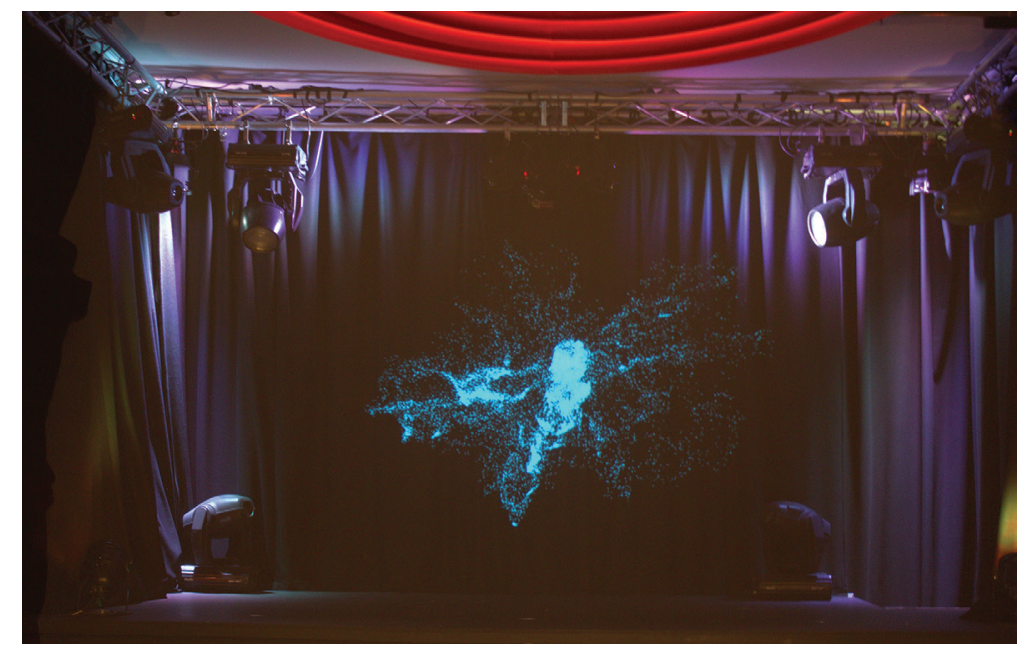

Figure 3. Transmission, 2014. Holographic Projection Test. $5 \mathrm{~m}$ x 2.8m using Pepper's Ghost display. December 2013. Transmission stands in a long tradition of EEG interpretation ranging back to the 1960 s and 1970s. $\odot 2014$ Analema Group.

the subject matter of academic research, they become artists themselves. Users learn about their own reactions to interactions and listen to their inner processes.

\section{Research}

Transmission presents a standardized model for the analysis of telepresence. Classification systems, models, and research parameters for telepresence have been conceptualized in many different ways over more than 30 years of academic discourse. As a result, key coherences across these various models have emerged. The focus in academic debate on telepresence as a terminus technicus has shifted gradually from its original, techno-centric conception towards a perceptionfocused, user-centric conception. Initial definitions linked the term to tele-robotics, tele-operators, and a man-machine discourse. These concepts were substituted by a discourse on virtuality and mediacy in the 1990s, and by the notions of perceived non-mediation, media irrelevance, and remote presence which dominate present-day discourse.

On a more analytical, methodological level, classifications for telepresence have been developed for sonic and visual telepresence, with various degrees of complexity. Renaud lists three distinctive cues for sonic telepresence: behavioral, notational, and temporal cues [28]. Lombard and Ditton [29] enumerate core components of telepresence such as social experience, realism, immersion, and transportation, among others. Witmer and Singer [30] differentiate between four distinctive factors: degree of control, sensory factors, distraction, and realism. Sadowski and Stanney [3I] identify individual and system variables such as ease of interaction, user-initiated control, pictorial realism, length of exposure, social factors, and system. Sheridan [32] identified five factors of telepresence- three technological and two context-based. Freeman favors a further reduction of discourse-relevant components to at most three factors: spatial physical presence, immersion, and social realism [33]. A strong argument for a simplified and coherent model of telepresence persists.

With Transmission, we narrow recurring discursive factors down to immersion, involvement, and realism. Immersion was singled out specifically by Barfield and Weghorst [34]. Prothero and Hoffman [35] and Hoffman, Prothero, Wells, and Groen [36] discussed the relevance of involvement; and Barfield and Hendrix focused on realism [37] as core elements of presence. Transmission is an ideal pilot to test such a comprehensive model. Analyzing a complex phenomenon over elemental key dimensions allows for a streamlined methodological approach. 
For a multi-sensory user-specific phenomenon, we require a conceptualization that ensures cross-platform compatibility, flexibility in application, and the possibility to be applied to both quantitative and qualitative research.

Telepresence has long left the confinements of academic discourse behind, to become a function of everyday life: people around the world engage in voice-over-Internet-protocol (VoIP) calls, videoconferencing, and Skype calls via handsets, tablets, laptops, etc. As the use of telepresence gains momentum across the globe, the need to understand the concept of remote presence increases. With a new plurality of media, new models for cross-platform analysis are required. With Transmission, we synthesize and condense existing strands of analysis into a succinct, standardized model. Our classification system is user-centric and focuses on individual experiences within the analysis. Furthermore, the model is media-unspecific and can be applied to a range of technologies. Depending on the choice of media, certain factors might be more or less conducive or detrimental to the overall perception of presence.

${ }_{3} \mathrm{D}$ environments, $3 \mathrm{D}$ display technologies, and augmented reality tools such as Oculus Rift [38] and Pepper's Ghost mediate presence as much as networked streaming, mobile phones, or tablets. Technical modalities and tools remain secondary in dominating definitions. Telepresence was defined by the International Society for Presence Research (ISPR) as "a psychological state or subjective perception in which, even though part or all of an individual's current experience is generated by and/or filtered through human-made technology, part or all of the individual's perception fails to accurately acknowledge the role of the technology in the experience" [39].

The focus on perceived non-mediation shifts the onus from the media onto the user. Presence is a multi-sensory communication experience of perceived non-mediation with immersive qualities. In conceptualizing immersion, realism, and interactivity as core factors of telepresence, we can look at their underlying sub-components and measure them individually.

Realism is often understood as a main component of presence. Image quality, transmission signal, lighting, etc. all affect how we perceive a transmitted image. Like immersion and interactivity, realism is a component of, but not a requirement for, presence. An abstract visual image can convey presence as much as a photorealistic video stream. Realism can be analyzed across subjective/perceptual parameters as well as technical parameters.

Equally, immersion is understood as both a perceptual/psychological reality and a technical condition. Immersion describes the subjective absorption into a mediated environment. The user's segregation from the outer world engenders a perceptual predominance of the media reality. Immersion can be measured through technical cues and subjective perceptual parameters.

The Oxford English Dictionary defines interactivity as a two-way flow of information between a computer and a user, allowing for the user's input [40]. Interactivity is a technical condition and a perceptive reality at once, a social condition and a state of involvement. As a co-factor for perceived presence, interactivity is generated through both technical and perceptual cues. The concept of interactivity is strongly related to concepts of intimacy and immediacy [4I]. As a technical condition, interactivity has been analyzed by Shrum and Liu [42]. Over the course of this project, we have measured technical factors as much as perceptual cues.

With Transmission, we analyze telepresence as a subjective (immersion) and social (interactivity) experience. Transmission operates in a dialectic field between individual perception and social 
activity, being introspective and interactive at the same time. EEG impulses are assembled as quantifiable data and compared to the qualitative data of questionnaires. Triangulation of quantitative EEG data with correlating qualitative tests (Figure 4) allows for a multidimensional analysis across various key factors [43]. Our research tackles the question of how immersion and interactivity can be related to telepresence. More than creating a pool of data, the aim is to communicate results of our analysis to the audience in situ. Users thus explore a complex phenomenon through interaction. Consolidating different conceptual angles, a simplified model of telepresence leads to new methods for crossplatform analysis.

\section{Technical Setup}

Transmission uses two main spaces: a motion capture studio to capture the movement of the user and a projection and display environment in which the user generates neurofeedback for visual and sonic representation.

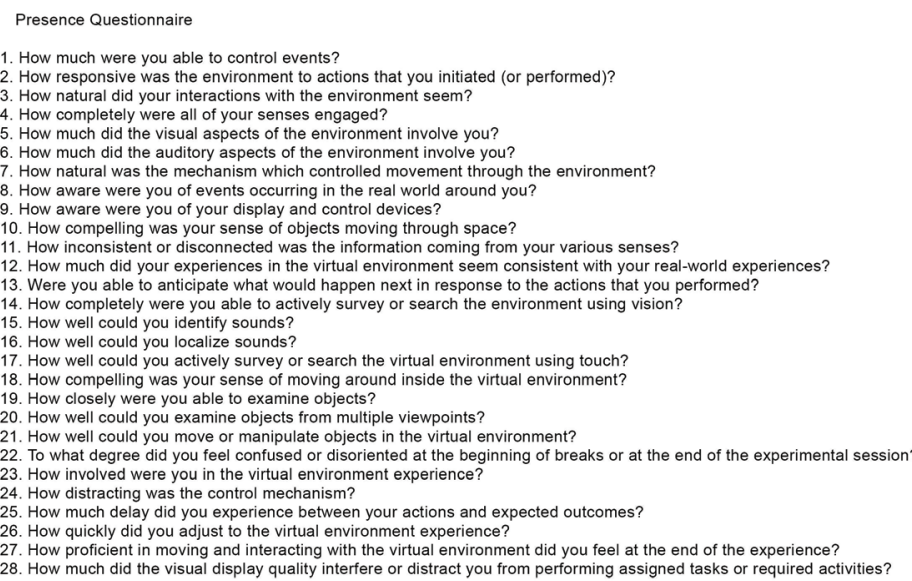

Figure 4. PQ (Presence Questionnaire), as developed by Witmer and Singer, will be distributed as part of the research on Transmission. Qualitative questionnaires will be compared to quantitative data resulting from EEG tests.

\section{MUSION}
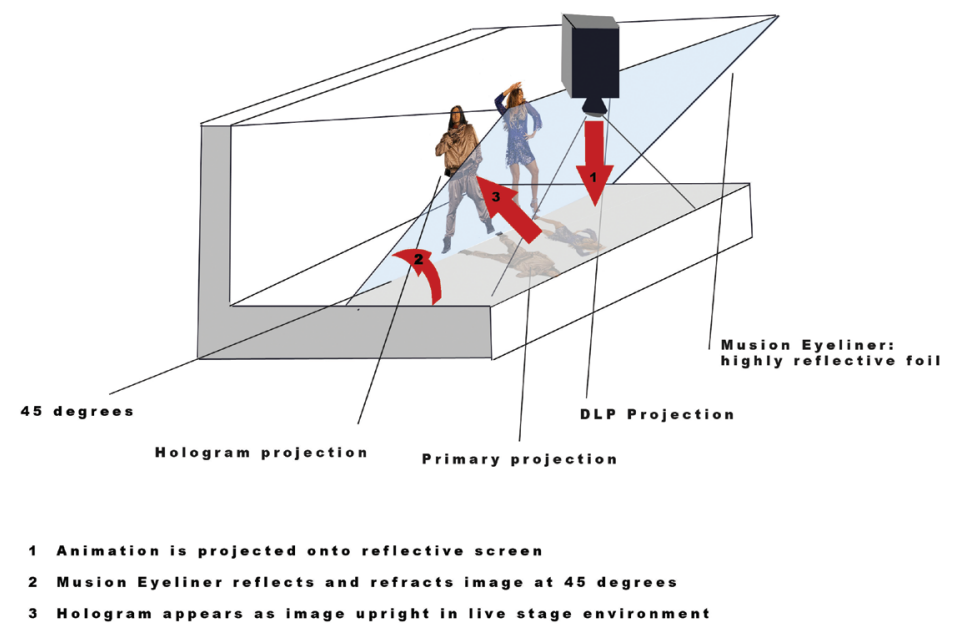

Figure 5. Schematic of a large-scale Pepper's Ghost projection. A holographic image is projected onto a live stage environment, allowing for real-time interaction between a virtual image and a live performer or user. () 2014 Musion.

Transmission's technical setup consists of a networked installation between the EEG and its visual interpretation output, a motion capture studio, and the projection environment. Motion data manipulates a particle stream displayed on a screen interface, such as a "holographic" (Pepper's Ghost) display in a remote location. Holographic screens will be preferred wherever practical. Sound, visual data, and motion data are generated and streamed in real time, allowing for their immediate alteration, and thus creating a constant feedback loop between external motion and internal fluctuation.

For testing purposes, we have linked a motion capture studio at Bournemouth University to a holographic projection studio in Central London (Musion) where the audience can observe and interact with an illustration of their own neural activity on a "holographic interface." Motion 
control systems were tested on the holographic screen interface as an on-site installation and in combination with telepresence. Recording and display parameters such as frame rate, streaming method, and camera angles were identified for recording and display environments.

Large-scale Pepper's Ghost displays (Figure 5) allow for the presentation of $3 \mathrm{D}$ images within a spatial environment via optical cues and a dedicated lighting environment. Holographic interfaces convey a certain cinematic, almost immersive, experience, which supports our goal for enhanced social engagement.
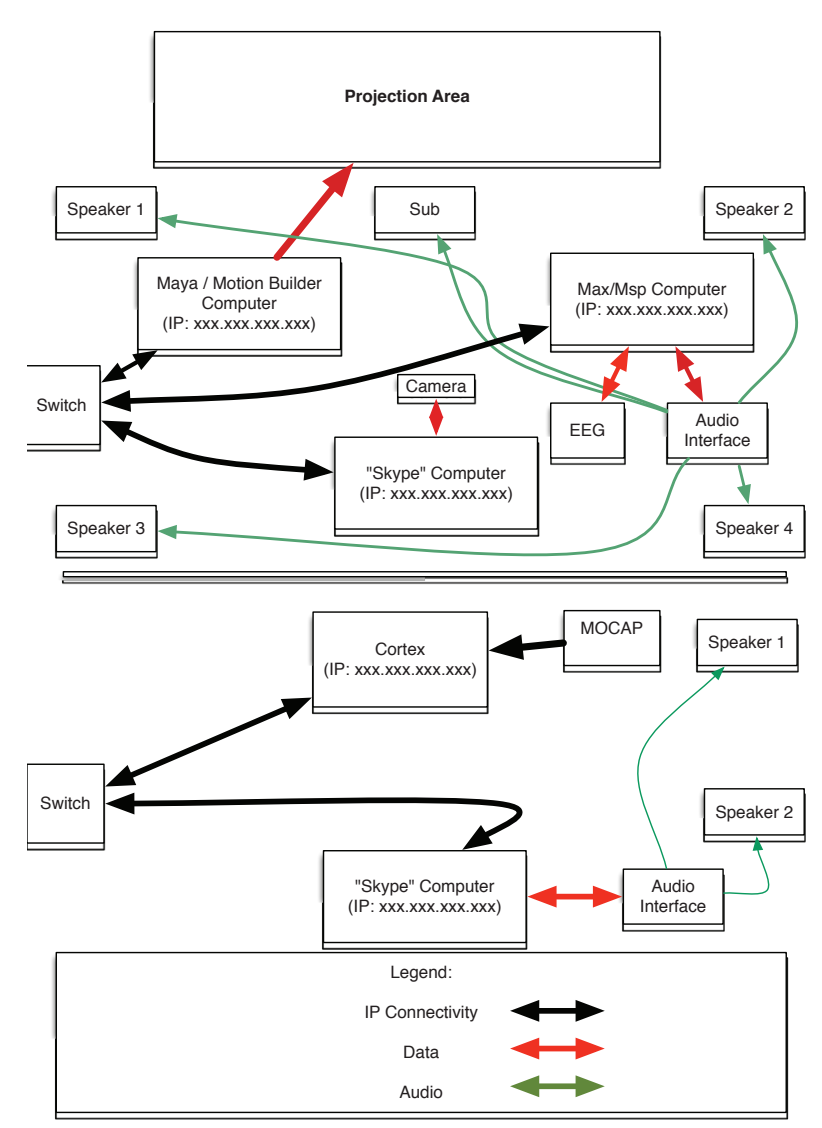

Figure 6. Schematic of Transmission setup. The setup is split between two spaces, one for EEG recording and one for remote motion capture. () 2014 Analema Group.
Brain wave data generated by the user are sonified in real time around the holographic stage area using multichannel speaker diffusion. The resulting immersive character of the soundscape corresponds to the visual qualities of the interactive display. A feedback signal is relayed from the holographic stage to the remote performer in the motion control studio. We have tested minimum motion control data transfer with a threshold of 6-8mbps upload and download speed. Higher bit rates allow for a more seamless experience of motion data streaming.

The Motion Analysis Cortex server parses the motion data from the motion capture control studio to the stage environment. In the future, other scenarios using Kinect motion capture or similar tools are planned. Data received from the Cortex server is passed onto Autodesk Maya via OSC (Open Sound Control) signals in real time through a network. The particle stream is based on parameters of the OSC signal input.

The effect stream is created as a real-time particle system within Maya using an OSC Maya plugin and OSC messages from the EEG device (emotiv epoc) [44]. The emotiv engine interprets the user's EEG data, passing them on as arguments of affective states. These emotional states are mapped onto colors and particle emissions of the particle system. A constant stream of particles visually represents the users' mental activity, providing them with information on their inner processes during the experience of telepresence. This real-time generated image is supported by a specially developed audio script that maps neuro-data into sound parameters for both interlocutors. Parameters such as specific changes in neural frequency oscillations, expressive or affective states can be mapped to sound signals with the aim of exploring certain communicational tendencies. Visual imagery is manipulated in real time by the user in a remote location. The generated imagery, as well as the EEG data, can be monitored for later analysis. 
In Transmission, neurofeedback methods are re-contextualized artistically, giving participants tools for interpretation of their own neural activity. A biofeedback loop enables us to extract visual mirror images of telepresence. On a sonic level, it allows the user to control and manipulate the soundscape in two locations through their actions, through focused attention or meditation and relaxation. Whereas visually, users control the representation of their neural activity through color codes and particle intensity, they are also immersed in a sound environment generated by their own brain activity. This procedural sound field is characterized by gradual discrepancies and changes in the EEG data input. Rather than neurofeedback signals, which consist of beep tones in a medical or therapeutic context, users experience their inner soundscape as a pleasant, continuous, and meditative surrounding. In Transmission, "sonic events," such as sound bursts and gentle vibrations, structure the experience and enforce a clear indication of the type of brainwave activity, e.g. relaxation, meditation, engagement, etc.

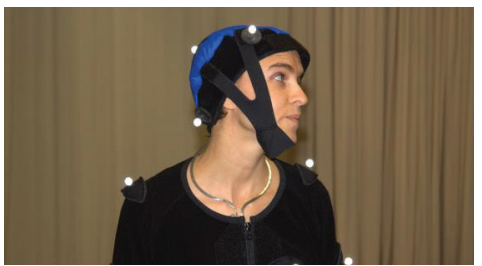

Figure 7. Analema Group's Eugenia Emets at the motion capture test. Multiple motion capture tests have been conducted. () 2014 Analema Group.
To create this feedback loop of actions and interactions, we send a reference signal to the motion data studio (Figure 6). The installation does not require extremely high bandwidth, as data interpretation is handled on-site. Only motion data and reference signals are streamed in real time.

A number of tests for sound, vision, and motion capture data [45] have been conducted to guarantee technical feasibility. Current tests at the Motion Analysis motion capture studio at Bournemouth University rely on a Cortex server (Figure

7). We tested OSC compatibility with Maya and software integration between Maya and Motion Analysis. An ongoing discourse with programmer Paolo Emilio Selva helps us to adapt OSC input into the Maya script. OSC data interpretation from the EEG headset was tested.

A multitude of tests between Geneva and London focused on EEG sonification-to help us understand latency implications and to develop a cohesive workflow. After comparison between different EEG systems, the choice fell on a flexible unit that has been successfully used in artistic contexts of similar scope. Transmission is technically, conceptually, sonically, and visually past its pilot stage. We are now in a position to optimize the workflow, present the project to the public, and concentrate on research design, data acquisition, and data interpretation.

\section{Research Design}

With our research, we are interested in generating tangible results-a set of viable, reliable, and representative data that reflects both the subjective, psychological as well as the objective, quantifiable aspects of telepresence. We can measure components of telepresence, such as interactivity and feelings of immersion, both directly through questionnaires, and indirectly by looking at factors such as time, affective EEG cues, and intensity of signal levels. With both standardized questionnaires (PQ) and a corollary set of EEG data at hand, we aim for comprehensive research results. Specifically, we want to find answers to the following questions:

- What affects the propensity to interact?

- How relevant is realism for the conveyance of presence?

- Is there a discrepancy between perceived and measured perceptions of presence?

- Is there a correlation between interactivity, immersion, and telepresence?

A public display is currently planned for the Bl-nk space in London at the end of 2014, in conjunction with Kinetica and Hackney Council. This installment of Transmission will be 
followed up with questionnaires and further analysis of the generated data. The research collective is working closely with a neuroscientist and a neurofeedback therapy practitioner, who will be closely involved in the interpretation of our research outcome.

\section{Future Outlook}

In addition to the conceptualization and production of Transmission as a participatory performance piece, we want to optimize the work as a user-focused installation. In the first instance, Transmission has been developed as a performance tool, involving professional choreographers and dancers in real-time interaction. A stand-alone installation piece will be developed as an audience-participation tool in a second phase. Sound modulation and visual representation will give users live insight into their brainwave frequency data while they communicate across two different spaces. A single-user mode as a more introspective, more educational device is also planned. Initial limitations on complexity of mapping and complexity of representation will be challenged in due course.

Visualization projects on human brain activity and the nervous system, such as The Human Connectome Project [46] or The Human Brain Project [47] lead the field in visualization strategies on the human brain. On an academic level, Transmission first and foremost concerns telepresence itself-as a cultural, a phenomenological, and a perceptual concept. On a conceptual level, Transmission is concerned with understanding and transcending the body-mind dichotomy. The body as a networked environment, as a network itself, a rhizome, a field of synapses and neural transmissions, is never self-enclosed, always interacting, reacting, emitting, and communicating. Transmission is intended to raise awareness for conscious reactions and interactions between the mind and the body.

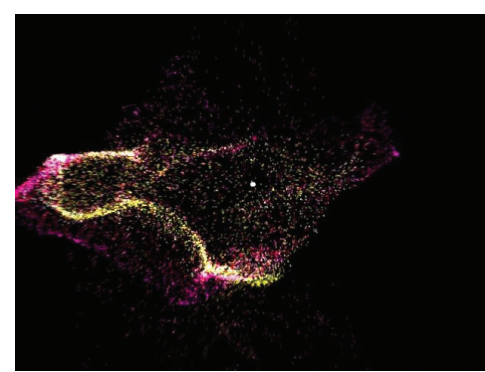

Figure 8. Transmission visualization. Screenshot 1920 x 1080, December 2013. With Transmission, Analema Group created a new interface for audiovisual interpretation of brain waves. The long-term plan is to find therapeutic and potentially even medical uses for this piece. @ 2014 Analema Group.

Transmission, once widely trialed and tested, will propose a technical infrastructure which could be used for medical and/or therapeutic purposes. Working closely with neurofeedback practitioners, we understand the need for more complex, visually and sonically developed interfaces for the interpretation of neural signals (Figure 8). The experience of the team and expert consultancy will ensure successful re-contextualization of our analysis tool. Ultimately, our objective is to introduce our interface into a therapeutic setting by adapting an artistic tool to practical considerations.

Academic research in the framework of this project combines standardized tests with quantifiable EEG data and subjective telepresence data with objective data sets. With the presentation of a standard model for telepresence, we want to focus on underlying factors and components of presence. Concentrating on three core factors-interactivity, immersion, and realism-we will discuss their interrelations, their relevance, and their characteristics within the research environment of Transmission.

On an artistic level, Transmission presents a new interface for reflective engagement with one another, a performance tool that integrates audiences by empowering them to "see" and explore their mind, their reflexes, their reactions and their interactions in a new light. The dialectic between inner evolutions and outer influences, mind and body, external and internal environments, is represented as a Mobius loop of infinite possibilities. 


\section{References and Notes}

I. Minsky, Marvin, “Telepresence,” Omni, 45-5I (1980).

2. Widely regarded as the first example of telepresence art, Roy Ascott's La Plissure $d u$ Texte was first exhibited at the Musée de l'Art de la ville de Paris. La Plissure du Texte, <http://alien.mur.at/rax/ ARTEX/PLISSURE/plissure.html> (Paris, I983), accessed March 24, 20I4. Mixed media.

3. Sermon, Paul, Telematic Dreaming, <www.paulsermon.org/> (Finland, 1992), accessed February I5, 20I4. Mixed media telepresence projection, variable dimensions.

4. Sermon, Paul, Telematic Séance, <www.paulsermon.org/> (Finland, 1993), accessed February I5, 2014. Mixed media, variable dimensions, telepresence projection.

5. Sermon, Paul, Telematic Vision, <www.paulsermon.org/> (Karlsruhe, 1994), accessed February I5, 20I4. Mixed media, variable dimensions.

6. Keane, Tina, Couch, <www.luxonline.org.uk/artists/tina_keane/couch.html> (multiple locations, 1998-2003), accessed January 5, 20I4. Mixed media, monitors, telepresence environment, and couch. Variable dimensions.

7. Lozano-Hemmer, Rafael, The Trace, <www.lozano-hemmer.com/the_trace.php> (I995), accessed March I7, 20I4. Outdoor projection. In Christiane Paul, Digital Art (London: Thames \& Hudson, 2003), $72-77$.

8. Lozano-Hemmer, Rafael, Displaced Emperors (Ars Electronica, Linz, 1998). Outdoor projection. In Christiane Paul, Digital Art (London: Thames \& Hudson, 2003), 72-77.

9. A good example for discourse on virtuality is Ken Goldberg's Telegarden, displayed at Ars Electronica Center (Linz, 1995-2004), <http://goldberg.berkeley.edu/garden/Ars/>, accessed March 15, 2014.

Io. Hayles, Katherine N., How We Became Posthuman (Chicago: Chicago University Press, I999).

II. Stelarc, Rotating Brains / Beating Heart, <www.youtube.com/watch?v=m36PRHsX $57 \mathrm{w}>$, accessed January 2, 20I4. A virtual-reality performance featuring Pauline Oliveros, Stelarc, and the Avatar Orchestra Metaverse (New Adventures in Sound Art SOUNDplay Festival, November 20I0, OCAD University, Toronto, Canada).

I2. Stanza, Connecting Cities, <www.stanza.co.uk/data/index.html>, accessed January I, 20I4. Live data visualization projection, Liverpool, 20Io.

13. Duchamp, Marcel, Bicycle Wheel, <www.moma.org/collection/object.php?object_id=8163I > (New York, 1913), accessed January 2I, 20I3. Mixed media, metal wheel mounted on painted wood stool. Dimensions: $129.5 \times 63.5 \times 41.9 \mathrm{~cm}$.

I4. Lieberman, Zach, et al., Eyewriter interactive read/writing software, <www.eyewriter.org/> (New York, 20IO), accessed February I2, 2014.

15. rAndom International, Rain Room, <http://random-international.com/work/rainroom/> (Barbican, London, 20I2), accessed February I6, 20I4. Water, injection-moulded tiles, solenoid valves, pressure regulators, custom software, $3 \mathrm{D}$ tracking cameras, steel beams, water management system, grated floor. Ioo sq $\mathrm{m}$.

I6. Paul, Christiane, Digital Art (London: Thames \& Hudson, 2003) 67.

17. Oliveros, Pauline, Deep Listening, <http://deeplistening.org/site/content/pauline-oliveros> (2009), accessed December 2I, 20I3.

18. Lucier, Alvin, Music for a Solo Performer, live performance, Brandeis University, 1965.

19. Teitelbaum, Richard, Spacecraft, <www.alvincurran.com/writings/mev.html> (New York, I967), accessed March 2I, 20I4. EEG-triggered live musical performance.

20. Rosenboom, David, Biofeedback and the Arts: Results of Early Experiments (Vancouver: Aesthetic Research Centre of Canada, 1974).

2I. Miranda, Eduardo, Thinking Music (Plymouth, UK: University of Plymouth Press, 2014).

22. Brouse, Andrew, Biomusic (Montréal: Society for Arts and Technology, 2007).

23. Abramovic, Marina, The Magic of Mutual Gaze, <www.youtube.com/watch?v=UtgoPo8sLJw $>$ (New York, 20II), accessed February 2I, 2014.

24. Cathode, Neam, Cyber Mondrian (Oboro Gallery, Montréal, 200I). EEG Performance. 
25. Mori, Mariko, Wave UFO <www.deitch.com/artists/sub.php?artistId=I5> (New York, 2003), accessed March I, 20I4.

26. Park, Lisa, Euonia <www.thelisapark.com/> (2013), accessed March 22, 2013.

27. Thompson, Jeffrey D., "Methods for Stimulation of Brainwave Function Using Sound," <www. neuroacoustic.com/methods.html> (I990), accessed March I3, 2014.

28. Renaud, Alain, "Dynamic Cues for Network Music Interactions," Proceedings of the SMC Conference 20I0, <http://smcnetwork.org/resources/smc2OIO>, accessed March 20, 2014.

29. Lombard, Matthew, and Theresa Ditton, “At the Heart of It All: The Concept of Presence," Journal of Computer-Mediated Communication 3, I083-610I (1997)

30. Witmer, Bob, and Michael Singer, "Measuring Presence in Virtual Environments: A Presence Questionnaire," Presence Vol. 7, No. 3, 225-240 (June 1998).

31. Sadowski, Wallace, and Kay Stanney, Measuring and Managing Presence in Virtual Environments (Orlando, FL: University of Central Florida, 2002), <http://web.cs.wpi.edu/_gogo/courses/imgd5Ioo/ papers/Sadowski_HVE_2002.html>, accessed March 20, 2014.

32. Sheridan, Thomas, "Musings on Telepresence and Virtual Presence," Presence Vol. I, No. I, I2O-I26 (1992).

33. Freeman, Jonathan, "Implications for the Measurement of Presence from Convergent Evidence on the Structure of Presence," paper presented to the Information Systems Division at the Annual Meeting of the International Communication Association (New Orleans, LA, 2004). In Campanella Bracken, Cheryl, and Paul Skalski, “Telepresence and Video Games: The Impact of Image Quality,” PsychNology Journal Vol. 7, No. I, IOI-II2 (2009).

34. Barfield, Woodrow, and Suzanne Weghorst, “The Sense of Presence Within Virtual Environments: A Conceptual Framework," Human-Computer Interaction: Software and Hardware Interfaces Vol B, 699-704 (1993).

35. Prothero, J.D., and H.G. Hoffman, "Widening the Field-of-View Increases the Sense of Presence in Immersive Virtual Environments," Human-Computer Interaction: HCI Intelligent Multimodal Interaction Environments, Vol. 4552, 393-400 (2007).

36. Hoffman, H.G., et al., "Virtual Chess: The Role of Meaning in the Sensation of Presence," International Journal of Human-Computer Interaction Vol. I0, 25I-263 (1998).

37. Barfield, Woodrow, and Claudia M. Hendrix, "The Effect of Update Rate on the Sense of Presence within Virtual Environments," Virtual Reality: The Journal of the Virtual Reality Society Vol. I, No. I, 3-16 (1995).

38. Oculus Rift, <www.oculusrift.com>, accessed March 2I, 2014.

39. International Society for Presence Research, "The Concept of Presence: Explication Statement," $<$ http://ispr.info> (2000), accessed February I5, 2014

40. Oxford English Dictionary, <www.oxforddictionaries.com/definition/english/interactive>, accessed January 4, 20I4.

4I. Lombard, Matthew, and Theresa Ditton, “At the Heart of It All: The Concept of Presence," Journal of Computer-Mediated Communication Vol. 3, I083-6IoI (1997).

42. Liu, Yuping, and Shrum, L.J., "What Is Interactivity and Is It Always Such a Good Thing? Implications of Definition, Person, and Situation for the Influence of Interactivity on Advertising Effectiveness," Journal of Advertising Vol. 4, 53-64 (2002).

43. Witmer, Bob, and Michael Singer, "Measuring Presence in Virtual Environments: A Presence Questionnaire," Presence Vol. 7, No. 3, 225-240 (June i998).

44. Emotiv EEG device, <www.emotiv.com>, accessed March 20, 2014.

45. Zhidong, Xiao, Motion Capture Based Motion Analysis and Motion Synthesis for Human-like Character Animation (Bournemouth University, 2009).

46. Human Connectome Project, <www.humanconnectomeproject.org>, accessed February 2I, 2014. 47. Human Brain Project, <www.humanbrainproject.eu>, accessed February I5, 2014. 